\title{
Hallazgos clínicos, ecocardiográficos y electrocardiográficos en donantes de sangre seropositivos para Trypanosoma cruzien un centro de referencia del sur de Colombia
} Clinical, cardiac ultrasonography, and electrocardiographic findings in blood donors who were found to be seropositive for Trypanosoma cruzi infection in a reference center in southern Colombia

Correspondencia

Cristhian Felipe Ramírez-Ramos cfrr89@gmail.com

Recibido: 27/03/2017

Arbitrado por pares

Aprobado: 07/06/2017

Citar como: Diaz-Cardozo EM, Motta-Quimbaya OJ, GiraldoBahamon G, Duran-Gutierrez LF, Polania-Segovia MP, RamírezRamos CF, et al. Hallazgos clínicos, ecocardiográficos y electrocardiográficos en donantes de sangre seropositivos para Trypanosoma cruzi en un centro de referencia del sur de Colombia. Acta Med Peru. 2017;34(3):188-95
Eduard Mauricio Diaz-Cardozo 1,2,3, Orfa Yaneth Motta-Quimbaya 2,3, German Giraldo-Bahamon 2,3, Luis Fernando Duran-Gutierrez ${ }^{2,3}$, Maria Paula PolaniaSegovia4, Cristhian Felipe Ramírez-Ramos ${ }^{2,3}$, Alvaro Mondragón-Cardona ${ }^{2,3}$

1 Departamento de cardiología, Fundación Cardioinfantil-Instituto de cardiología. Universidad el Bosque, Bogotá-Colombia.

2 Departamento de Medicina Interna, Universidad Surcolombiana, Hospital Universitario Hernando Moncaleano Perdomo, Neiva, Huila Colombia.

3 Grupo de investigación MI-Dneuropsy, semillero de investigación medicina interna SIMI, Universidad Surcolombiana, Neiva, Huila Colombia.

4 Fundación Universitaria Juan N Corpas, Bogotá-Colombia.

\section{RESUMEN}

Objetivo: Evaluar las características clínicas y hallazgos electrocardiográficos como de ecocardiografía en pacientes quienes tenían una serología positiva en el banco de sangre de un centro de referencia del sur de Colombia. Material y métodos: Estudio descriptivo, de corte transversal, en el que se analizaron todos los eventos de donación del banco de sangre del Hospital Universitario Hernando Moncaleano Perdomo, de Neiva Huila Colombia, en un periodo comprendido entre enero del 2011 hasta diciembre de 2014. Fueron incluidos pacientes mayores de 18 años, con serología IgG por ELISA positiva para Trypanosoma cruzi, posteriormente confirmada por detección de IgG por inmunofluorecencia indirecta. Se les realizó anamnesis clínica, examen físico, ecocardiogramas transtorácicos y monitoreos electrocardiográficos de ritmo cardiaco 48 durante horas. Resultados: Se registraron 41865 donantes en el banco de sangre, la prevalencia de infección por T. cruzi fue de $0,15 \%$, promedio de edad 47,8 años, $68,42 \%$ de género masculino y la mayoría de zona urbana. Un porcentaje importante de los pacientes manifestó alguna sintomatología relacionada con cardiopátia chagásica. Además la mayoría presentó compromiso de la fracción de inyección del ventrículo izquierdo (leve a moderado) y en la totalidad se detectó alguna anormalidad electrocardiográfica. Conclusión: El hallazgo en el índice de variabilidad de la frecuencia cardíaca se debería considerar como un posible indicador importante en esta patología, en cuanto al reconocimiento temprano antes de notar alteraciones estructurales que generen una falla cardíaca sintomática en esta patología.

Palabras clave: Enfermedad de Chagas; Cardiomiopatía Chagásica; Donantes de sangre; Ecocardiografía; Electrocardiografía ambulatoria (fuente: DeCS BIREME). 


\section{ABSTRACT}

Objective: This study aimed to assess the clinical characteristics and the electrocardiographic and cardiac ultrasonography findings in patients who had positive serology test results for Chagas' disease in a reference center in southern Colombia. Materials and methods: This is a descriptive, cross-sectional study in which we assessed all blood donating events in the blood bank of the Hernando Moncaleano-Perdomo Hospital in Neiva Huila, Colombia, from January, 2011 to December, 2014. Patients more than 18 years old who had positive results in the serum IgG measurement for Trypanosoma cruzi, which had to be confirmed afterwards using indirect immunofluorescence for IgG detection. The patients had a medical history taken, they underwent physical examination, transthoracic cardiac ultrasonography, and electrocardiographic continuous 48-hour monitoring. Results: Nearly forty-two thousand $(41,865)$ blood donors were recorded in the blood bank. The prevalence of $T$. cruzi infection was $0.15 \%$, the average age of the subjects was 47.8 years, two thirds (68.42\%) were male, and most came from urban areas. An important proportion of patients presented with some symptoms related to Chagas' disease cardiopathy, most had alterations in the left ventricular ejection fraction and all of them showed at least one electrocardiographic abnormality. The finding in the index of heart rate variability should be considered as a possible early indicator in this pathology, before the presence of structural alterations that could generate a symptomatic heart failure.

Keywords: Chagas disease; Chagas cardiomyopathy; Blood donors; Echocardiography; Electrocardiography, ambulatory (source: MeSH NLM).

\section{INTRODUCCIÓN}

La infección por Trypanosoma cruzi, causa de la enfermedad de Chagas, es aún un importante problema de salud pública ${ }^{[1,2]}$. A pesar de su descubrimiento "reciente" [3], se cree que esta zoonosis parasitaria existe de forma natural en el continente americano desde hace más de 9000 años, se ha documentado que pobladores de comunidades prehistóricas del norte de Chile y el sur del Perú habrían sufrido de infección chagásica ${ }^{[4]}$.

Para el año 2008, cerca de 8 millones de personas en Latinoamérica presentaron la infección, que provocó de 10000 a 14000 muertes al año, además que, según aproximaciones de la Organización Panamericana de la Salud (OPS), el 20\% de la población estaría en riesgo ${ }^{[5,6]}$. Para el año 2010 se estimaron 5742167 nuevos casos ${ }^{[7]}$. En Colombia, para el mismo año, la Organización Mundial de la Salud indicó que alrededor de 437960 personas estaban infectadas, con un cálculo de 5274 casos nuevos al año ${ }^{[7]}$.

La evolución clínica de esta entidad incluye tres fases: aguda, indeterminada y crónica. Estas fases, hacen parte de un complejo sintomático mal definido y poco específico. La fase inicial o aguda es frecuentemente asintomática; sin embargo, de presentarse, puede ser similar a cualquier entidad viral. La fase indeterminada también es asintomática. En la fase crónica se presentan diferentes manifestaciones clínicas como la demencia (3\%), cardiomiopatía (30\%) y dilatación del tracto digestivo (6\%) ${ }^{[8]}$.

Durante la miocardiopatía por Chagas las arritmias son la principal manifestación siendo las extrasístoles ventriculares polimórficas un hallazgo común, al igual que el bloqueo completo de rama derecha, hemibloqueo anterior izquierdo, bloqueo auriculoventricular, y los cambios en la repolarización ventricular [9]. La mortalidad estimada por cardiopatía Chagásica es de 45 mil personas al año, siendo una de las condiciones patológicas con mayor índice de muerte súbita impredecible ${ }^{[10]}$.
Diversos estudios han documentado de manera clara las anormalidades electrocardiográficas en pacientes en la fase indeterminada, en pacientes con serologías positivas en bancos de sangre por diversos métodos. Además, han evaluado la evolución ecocardiográfica y progresión a enfermedad cardiaca sintomática, como también la respuesta al tratamiento en la fase indeterminada, evidenciando un efecto positivo del mismo en los distintos reportes ${ }^{[11-18]}$. El objeto del presente estudio fue evaluar las características clínicas y hallazgos electrocardiográficos como de ecocardiografía en pacientes quienes tenían una serología positiva en el banco de datos de un centro de referencia del sur de Colombia.

\section{MATERIALES Y MÉTODOS}

\section{Tipo de estudio}

Estudio descriptivo transversal. Se analizaron todos los eventos de donación del banco de sangre del Hospital Universitario Hernando Moncaleano Perdomo, de Neiva (442 m de altitud), departamento de Huila, ubicado al sur de Colombia, en un periodo de tiempo de cuatro años (enero de 2011 a diciembre de 2014), determinado por disponibilidad de registros electrónicos de evento de donación.

\section{Población y muestra}

Fueron incluidos la totalidad de eventos de donación desde el año 2011 hasta 2014, de los cuales fueron estudiados los pacientes que cumplieron con los criterios de inclusión.

\section{Criterios de selección}

Se incluyeron a aquellos pacientes mayores de 18 años, con serología IgG por ELISA positiva para Trypanosoma cruzi, posteriormente confirmada por detección de IgG por inmunofluorecencia indirecta positiva y que aceptaran participar 


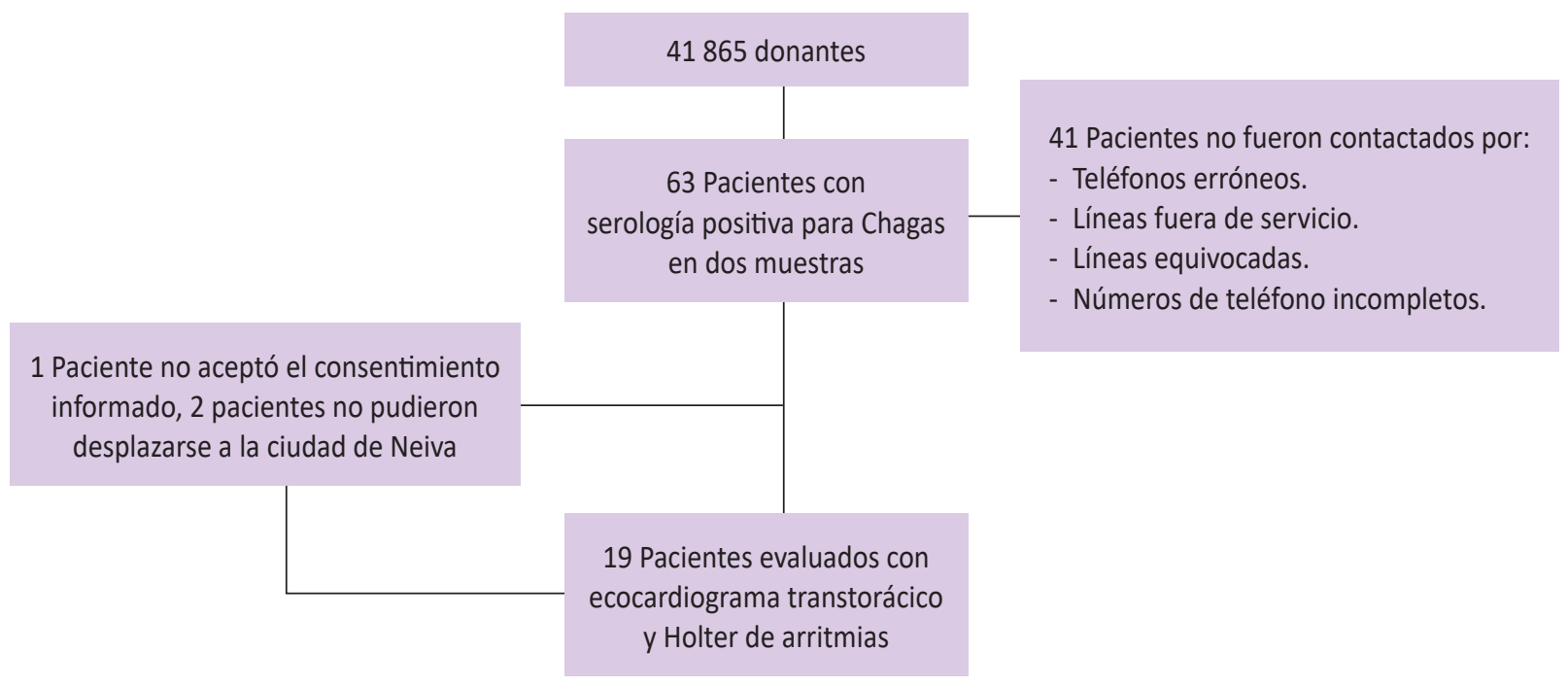

Figura 1. Proceso de selección de pacientes involucrados en el estudio.

en el estudio mediante el consentimiento informado y asistencia para recolección de datos clínicos y realización de pruebas paraclínicas. Se excluyeron a todos aquellos pacientes que no asistieran a las respectivas valoraciones clínicas y realización de monitoreo electrocardiográfico y ecocardiograma.

\section{Variables}

Los pacientes seleccionados fueron contactados telefónicamente para que, en una cita presencial, un médico especialista en medicina interna y cardiología realice la respectiva anamnesis clínica y examen físico. Además, se recolectaron, en el instrumento diseñado por los autores, datos sociodemográficos, antecedentes personales, variables clínicas básicas, antecedentes de contacto con el vector y búsqueda dirigida de signos clínicos de falla cardiaca o relacionados con la enfermedad. Además, se ejecutaron e interpretaron ecocardiogramas transtorácicos con equipo VIVID I, modelo H4502 1WP, con protocolo de 20 minutos y se instalaron los dispositivos de monitoreo electrocardiográfico de ritmo cardiaco durante 24 horas, empleando el equipo CardioScan modelo DMS 300-3.

\section{Análisis estadístico}

Se realizó estadística descriptiva con el respectivo análisis univariado. Para la caracterización de la población participante se utilizaron medidas de tendencia central y de dispersión. Los resultados obtenidos en el estudio fueron almacenados en una hoja de cálculo de Excel 2010 para Windows y analizados con el paquete estadístico SPSS versión 22.0.

\section{Aspectos éticos}

A nivel nacional según la resolución N8430 de 1993 del Ministerio de Salud de Colombia en su artículo 16 parágrafo primero, por tratarse de un estudio de intervención requirió la obtención del consentimiento informado el cual fue recolectado para la totalidad de los participantes, las intervenciones realizadas se consideran de bajo riesgo y la totalidad de los pacientes fueron informados de los resultados y direccionados a seguimiento médico especializado. Además, el presente estudio fue aprobado por el comité de ética médica del Hospital Universitario Hernando Moncaleano Perdomo mediante la resolución número 1756 de 2015.

\section{RESULTADOS}

Durante el periodo estudiado 63 presentaron serología IgG positivos para Trypanosoma cruzy por ELISA y confirmados por una segunda prueba de detección de IgG para Trypanosoma cruzy por método de inmunofluorecencia indirecta, correspondiendo a una proporción de $0,15 \%$ de un total de 41865 donantes en el banco de sangre (Figura 1). De estos se pudieron contactar y evaluar posteriormente 19 pacientes.

El promedio de edad fue de 47,8 años, con 13 donantes masculinos $(68,4 \%)$. Los pacientes procedían principalmente del departamento del Huila (73,6\%), lugar de ubicación del Banco de sangre, seguido de departamentos de Caquetá (10,5\%), Tolima $(10,5 \%)$ y Casanare $(5,3 \%)$, los que se encuentran dentro de la zona de influencia del hospital.

Se encuestó sobre el antecedente de haber presenciado la picadura del triatominios, solo cuatro pacientes manifestaron recordar la picadura del vector. Con respecto a los antecedentes médicos (reportados por los entrevistados), el más frecuente fue la dislipidemia $(31,5 \%)$, seguido por consumo de tabaco $(15,7 \%)$ e hipertensión arterial $(15,7 \%)$; ningún paciente indicó tener antecedentes personales de enfermedad coronaria y cardiopatía. Solo dos personas $(10,5 \%)$ indicaron haber recibido hemoderivados en alguna oportunidad. Respecto a antecedentes familiares, solo un paciente $(5,2 \%)$ manifestó historia muerte 
Tabla 1. Datos sociodemográficos y hallazgos a la anamnesis.

\section{Característica}

n

Género

Masculino

13

6

Femenino

14

2

Caquetá

Tolima

2

Casanare

Localización

Urbana

11

8

57,89

Rural

Escolaridad

Profesional

26,32

Básica secundaria

31,58

Básica primaria

31,58

Ninguna

10,53

Referencia picadura

Sí

No

Antecedentes personales

Hipertensión arteria

Dislipidemia

15,79

Consumo tabaco

Consumo alcohol

Transfusión sanguínea

3

31,58

15,79

5,26

10,53

Antecedentes familiares

Muerte súbita *

5,26

Cardiopatía *

1

5,26

Sintomatología

Palpitaciones

36,84

Disnea

47,37

Ortopnea

10,53

Precordialgia

42,11

Presincope

15,79

Edema

10,53

Disfagia

5,26

Estreñimiento

15,79

* En ambos casos correspondieron al hermano del paciente

súbita y un paciente $(5,2 \%)$ historia de cardiopatía en familiares de primer grado (hermano). Ocho pacientes no refirieron ningún tipo de antecedente.

Se evaluó la presencia de sintomatología relacionada con cardiopatía chagásica y falla cardiaca secundaria. La mayoría de los pacientes (68\%) manifestaron algún síntoma, siendo los más frecuentes disnea $(47,3 \%)$, dolor torácico $(42,1 \%)$ y palpitaciones (36,8\%); sin embargo, al evaluar la clasificación de la New York Heart Association (NYHA) todos se encontraban en clase funcional I. Los datos sociodemográficos y hallazgos a la anamnesis se resumen en la Tabla 1.

Los registros electrocardiográficos de 24 horas, mostraron alteraciones en la totalidad de los pacientes, siendo las extrasístoles supraventriculares $(89,4 \%)$ el hallazgo anormal más frecuente, seguido de las extrasístoles ventriculares (52,6\%). Se evaluó la variabilidad considerada como la desviación estándar de los intervalos entre los latidos normales (SDNN) y el índice triangular, que estiman la variabilidad promedio, encontrándose conservada en la mayoría de los casos (78,9\%). Sólo un paciente presentó taquicardia ventricular.

Con respecto al ecocardiograma transtorácico, se reportó dentro de límites normales en nueve pacientes. Los hallazgos anormales $(52,6 \%)$, estuvieron representados por: dilatación biauricular $(31,6 \%)$, dilatación ventricular derecha $(5,2 \%)$, dilatación ventricular izquierda tanto sistólica como diastólica (21\%), hipertrofia ventricular izquierda (10,5\%); se evidenció disfunción diastólica en nueve pacientes y alteraciones valvulares en 13 pacientes, siendo severa en un paciente $(5,2 \%$; insuficiencia mitral severa), relacionada a dilatación del anillo (de origen funcional). Considerando compromiso de la fracción de eyección a valores menores a 40\%, al revisar los reportes de ecocardiograma no se obtuvieron pacientes bajo este rango. Los hallazgos electrocardiográficos y ecocardiográficos se resumen en la Tabla 2.

Basados en la clasificación clínica de la cardiopatía chagásica ${ }^{[19,20]}$ (Tabla 3), el 73,7\% de los pacientes se encontraban en fase crónica indeterminada, el resto de pacientes se clasificó en la categoría B1, considerando los trastornos eléctricos encontrados en el registro electrocardiográfico de 24 horas. Se determinó el riesgo de muerte a 10 años por la escala de Rassi ${ }^{[21]}$ (que puntúa como bajo riesgo [0-6 puntos], riesgo intermedio [7-11 puntos] $y$ alto riesgo [12-20 puntos]); el $84,2 \%(n=16)$ de los pacientes tenía riesgo bajo (riesgo de muerte a los 5 años $2 \%$ y a los 10 años $10 \%$ ), el restante $15,7 \%$ se encontraba en la categoría de riesgo intermedio (riesgo de muerte a los 5 años $18 \%$ y a los 10 años 44\%).

\section{DISCUSIÓN}

La enfermedad de Chagas continúa siendo un importante problema de salud pública en Latinoamérica ${ }^{[7]}$. Porque esta infección es potencialmente transmisible por transfusión de hemoderivados, es importante conocer la prevalencia de dicha enfermedad en personas que aún no hayan desarrollado sintomatología debido a la propia historia natural ${ }^{[10]}$. En Latinoamérica diferentes estudios utilizando pruebas serológicas han encontrado prevalencias entre $0,05 \%{ }^{[14]}$ y $0,31 \%{ }^{[11]}$, resultados que son coherentes con lo encontrado en nuestro estudio. 
Tabla 2. Hallazgos electrocardiográficos y ecocardiográficos.

\begin{tabular}{|c|c|c|c|}
\hline Variable & Valor de referencia & $\mathbf{n}$ & $\%$ \\
\hline \multicolumn{4}{|l|}{ Hallazgos electrocardiográficos } \\
\hline Extrasistoles supraventriculares & & 17 & 89,47 \\
\hline Extrasistoles ventriculares & & 10 & 52,63 \\
\hline Taquicardia supraventricular & & 3 & 15,79 \\
\hline Fibrilación auricular & & 2 & 10,53 \\
\hline Taquicardia ventricular & & 1 & 5,26 \\
\hline Bloqueo Auriculo-Ventricular & & 2 & 10,53 \\
\hline \multicolumn{4}{|l|}{ Variabilidad electrocardiográfica } \\
\hline Conservada & & 15 & 78,95 \\
\hline Levemente comprometida & & 2 & 10,53 \\
\hline Moderadamente comprometida & & 2 & 10,53 \\
\hline Severamente comprometida & & 0 & 0,00 \\
\hline \multicolumn{4}{|l|}{ Ecocardiograma } \\
\hline $\mathrm{FEV} \mid>=40 \%$ & & 19 & 100,00 \\
\hline $\mathrm{FEVI}<=40 \%$ & & 0 & 0,00 \\
\hline Aurícula derecha dilatada & & 3 & 15,79 \\
\hline \multicolumn{4}{|l|}{ Aurícula izquierda- volumen } \\
\hline Normal & $<34 \mathrm{ml} / \mathrm{m}^{2}$ & 16 & 84,21 \\
\hline Leve & $34-41 \mathrm{ml} / \mathrm{m}^{2}$ & 0 & 0 \\
\hline Moderada & $42-48 \mathrm{ml} / \mathrm{m}^{2}$ & 3 & 15,79 \\
\hline Severa & $>48 \mathrm{ml} / \mathrm{m}^{2}$ & 0 & 0 \\
\hline Ventrículo izquierdo en sístole dilatado & & 2 & 10,53 \\
\hline Hombres & $11-31 \mathrm{ml} / \mathrm{m}^{2}$ & & \\
\hline Mujeres & $8-24 \mathrm{ml} / \mathrm{m}^{2}$ & & \\
\hline Ventrículo izquierdo en diástole dilatado & & 2 & 10,53 \\
\hline Hombres & $34-74 \mathrm{ml} / \mathrm{m}^{2}$ & & \\
\hline Mujeres & $29-61 \mathrm{ml} / \mathrm{m}^{2}$ & & \\
\hline Ventrículo derecho dilatado & & 1 & 5,26 \\
\hline Diámetro basal & $25-41 \mathrm{~mm}$ & & \\
\hline Diámetro medio & $19-35 \mathrm{~mm}$ & & \\
\hline Disfunción diastólica* & & 9 & 47,37 \\
\hline Grado 1 & & 7 & 36,8 \\
\hline Grado 2 & & 2 & 10,52 \\
\hline Grado 3 & & 0 & 0 \\
\hline Disfunción valvular & & 13 & 68,42 \\
\hline Insuficiencia tricuspidea leve† & & 8 & 42,11 \\
\hline Insuficiencia aórtica leveł & & 1 & 5,26 \\
\hline Insuficiencia mitral§ & & 4 & 21,05 \\
\hline Leve & & 3 & 15,78 \\
\hline Moderada & & 0 & 0 \\
\hline Severa & & 1 & 5,26 \\
\hline
\end{tabular}

* Clasificación de la disfunción diastólica: grado 1 (diámetro de la auricular izquierda normal o baja $<34 \mathrm{ml} / \mathrm{m}^{2}$, relación E/A $<0=0,8 \mathrm{~m} / \mathrm{seg}$, relación E/e $<10 \mathrm{~cm} / \mathrm{seg}$, pico de regurgitación tripuspidea $<2,8 \mathrm{~m} / \mathrm{seg}$ ); grado 2 (diámetro de la auricular izquierda $>34 \mathrm{ml} / \mathrm{m}^{2}$, relación $\mathrm{E} / \mathrm{A}>0,8<2,0 \mathrm{~m} / \mathrm{seg}$, relación $\mathrm{E} / \mathrm{e} 10-14 \mathrm{~cm} / \mathrm{seg}$, pico de regurgitación tripuspidea $>2,8 \mathrm{~m} / \mathrm{seg}$ ); Grado 3 (diámetro de la auricular izquierda $>34 \mathrm{ml} / \mathrm{m}^{2}$, relación E/A $>2,0 \mathrm{~m} / \mathrm{seg}$, relación E/e $>14 \mathrm{~cm} / \mathrm{seg}$, pico de regurgitación tripuspidea $>2,8 \mathrm{~m} / \mathrm{seg}$ ).

† Todos los pacientes presentaron insuficiencia tricuspidea leve: doppler color pequeño y central con una seña de CW débil; método semicuantitativo radio PISA $<5$ $\mathrm{mm}$; flujo de vena hepática con dominancia de onda S; métodos cuantitativos EROA $<40 \mathrm{~mm}^{2}$, con un volumen regurgitante menor a $45 \mathrm{ml}$.

₹ Todos los pacientes presentaron insuficiencia aórtica leve: paramentros cualitativos (yet color pequeño y central con un $\mathrm{CW}$ incompleto y débil sin flujo reverso diastólico en aorta descendente/aorta abdominal o protodiastólico; método semicuantitativo con ancho de vena contracta $<3 \mathrm{~mm}$, tiempo de hemipresión $>500$ mseg; método cuantitativo EROA $<10 \mathrm{~mm}^{2}$ y volumen regurgitante menor de $30 \mathrm{ml}$.

$\S$ Insuficiencia mitral: Leve: flujo color pequeño y central, señal del CW debil y parabólico; semicuantitativo vena contracta menor de $3 \mathrm{~mm}$, flujo en vena pulmonar con dominancia de la onda S, flujo mitral con dominancia de la onda A, VTI mitral/ VTI aortico $<1$; cuantitativo EROA $<20 \mathrm{~mm}^{2}$, volumen regurgitante $<30 \mathrm{ml}$. Moderada: flujo color intermedio, señal CW densa/ parabólica; semicuantitativo vena contracta menor de 4-7 mm, flujo en vena pulmonar con reducción de la onda S, flujo mitral variable, VTI mitral/VTI aórtico entre 1 y 1.4 ; cuantitativo EROA $20-40 \mathrm{~mm}^{2}$, volumen regurgitante $30-60 \mathrm{ml}$. Severo flujo color yet central grande, señal CW densa/ triangular; semicuantitativo vena contracta menor de $>7 \mathrm{~mm}$, flujo en vena pulmonar con inversión de la onda S, flujo mitral con onda E >1,5 m/seg, VTI mitral/ VTI aórtico $>1,4$; cuantitativo EROA $>40 \mathrm{~mm}^{2}$, volumen regurgitante $>60 \mathrm{ml}$. 
Tabla 3. Clasificación clínica de cardiopatía chagásica ${ }^{[20]}$.

Grupo clínico

Estado A (crónica indeterminada)

Estado B

B1

B2

Estado C

Estado D

Caracterización

Asintomática, no alteraciones significativas al examen físico, EKG, radiografía de tórax, esofagograma y enema con bario. No cambios en ecocardiograma, prueba de esfuerzo y Holter.

Paciente con enfermedad cardiaca estructural sin signos ni síntomas de falla cardiaca.

Pacientes con cambios en el EKG (arritmias o desórdenes de conducción) puede presentar leves anormalidades ecocardiográficas (alteraciones contractibilidad), con función global normal.

Pacientes con disfunción ventricular global (fracción de eyección disminuida).

Disfunción del ventrículo izquierdo y síntomas previos o actuales de falla cardiaca (NYHA I, II, III y IV)

Síntomas de falla cardiaca en reposo, refractaria a la terapia médica optimizada (NYHA IV) requiriendo intervenciones intensivas.

Fuente: Andrade JP, Marin-Neto JA, Paola AA, Vilas-Boas F, Oliveira GM, Bacal F, et al. [I Latin American guidelines for the diagnosis and treatment of Chagas cardiomyopathy] Arquivos brasileiros de cardiologia. 2011;97(2 Suppl 3):1-48.

Sin lugar a duda, el mayor impacto en morbilidad y mortalidad de la infección está dado por la cardiopatía chagásica ${ }^{[22]}$, y los pacientes pueden permanecer asintomáticos por un largo periodo de tiempo ${ }^{[23]}$, a pesar de la presencia de alteraciones estructurales y/o eléctricas a nivel cardiovascular [24], manifestando una clínica nutrida solo en etapas avanzadas de la enfermedad ${ }^{[25]}$.

En los pacientes seropositivos de nuestro estudio para Trypanosoma cruzi, son notables las alteraciones del monitoreo electrocardiográfico que se presentaron en la totalidad de la muestra, en contraste a otros estudios latinoamericanos donde se reportan tasas variables entre el $21,3 \%{ }^{[26]}$ y $11,5 \%{ }^{[27]}$ en áreas urbanas y entre $22 \%{ }^{[12]}$ y $40 \%$ en regiones rurales. En Colombia en el estudio CHICAMOCHA ${ }^{[15]}$ (Cardiovascular Health Investigation from Colombia to Assess the Markers and Outcomes of Chagas disease), en el cual se estudiaron 486 donantes de bancos de sangre seropositivos en la ciudad de Bucaramanga entre los años 1999 y 2004 , se encontraron alteraciones eléctricas en el 16,8\%.

Las principales alteraciones electrocardiográficas en el estudio fueron la presencia de extrasístoles supraventriculares y ventriculares, a diferencia de datos previos en los cuales las alteraciones más frecuentes en esta fase de la enfermedad (asinomatica) fueron los cambios inespecíficos de repolarización (33\%), seguidos por la bradicardia sinusal $(25 \%)$ cambios de la onda $\mathrm{T}(4,8 \%)$, el bloqueo incompleto de rama derecha en el $6,24 \%$ y el $6,1 \%$ bloqueo de rama derecha en el estudio colombiano ${ }^{[15,27]}$.

En los monitoreos electrocardiográficos se detectó la presencia de taquicardia supraventricular, taquicardia ventricular sostenida y de fibrilación auricular, las cuales no están descritas en los diferentes estudios publicados y cuyo reconocimiento es de vital importancia para definir una intervención temprana y de esta forma prevenir muerte súbita y complicaciones como eventos embólicos ${ }^{[15,16,18]}$.

La enfermedad de Chagas cursa con alteraciones tempranas del control autonómico de los reflejos cardiacos. Sin embargo, alteraciones en la dinámica no lineal de la variabilidad del intervalo RR no han sido estudiadas sistemáticamente $y$, como se señaló, se encontró alteración de la variabilidad de la frecuencia cardiaca en 4 de los 19 pacientes, dos de los cuales cursaron con un compromiso moderado. Se ha documentado una reducción progresiva de la frecuencia cardiaca y de la variabilidad concluyendo que existen alteraciones tempranas en la dinámica de la periodicidad del intervalo RR que preceden el compromiso miocárdico en pacientes con esta enfermedad ${ }^{[28]}$. Estos hallazgos pueden ser de utilidad para la identificación temprana de sujetos seropositivos que eventualmente desarrollarán cardiomiopatía chagásica y en quienes es importante tomar en cuenta esta variable como predictora de la evolución de la enfermedad o indicadora de seguimiento.

En cuanto a las alteraciones ecocardiográficas, en este grupo de pacientes se encontraron anomalías en 9 de 19 pacientes siendo la disfunción diastólica la principal; de los tipos de disfunción diastólica 8 de 9 pacientes cursaba con alteración en la relajación. Cuatro pacientes presentaron dilatación de cavidades, siendo concordante con resultados hallados en otros estudios que han evidenciado alteraciones ecocardiográficas en $38,8 \%$ siendo el agrandamiento de cavidades el hallazgo más frecuente (31\%) seguido de la alteración de la relajación ventricular $\left(27,6 \%{ }^{[18]}\right.$. En el mismo se documentaron en algunos casos alteraciones de la motilidad, no evidenciadas por los hallazgos en los pacientes estudiados, lo cual se sugiere está en relación con que estas se vinculan frecuentemente con etapas sintomáticas de la cardiopatía chagásica. Así mismo, la fracción de eyección se encontró conservada la totalidad de los pacientes al igual que en otros estudios con pacientes de iguales características ${ }^{[18,26]}$.

La realización de los estudios diagnósticos fue importante debido a la posibilidad de clasificar en el estadio clínico de la enfermedad: catorce pacientes presentaban la forma indeterminada y cinco pacientes en cardiopatía chagásica establecida. Esto se debe tener en cuenta, pues durante los últimos años se ha generado una gran discusión sobre la indicación del tratamiento en pacientes en fase indeterminada. Viotti et al. ${ }^{[14]}$ realizaron un estudio con el objetivo primario valorar la evolución a fase crónica avanzada 
de la enfermedad. Los resultados obtenidos evidenciaron que el $14,1 \%$ de los pacientes del grupo que no recibió tratamiento y el $4,2 \%$ en el grupo que si recibió respectivamente evolucionaron a etapas avanzadas de enfermedad. En cuanto la mortalidad, esta fue de $1,1 \%$ en el grupo tratado y $4,2 \%$ en el grupo no tratado. Esto género como recomendación que el tratamiento se debería ser ofrecido a pacientes adultos infectados sobre los 50 años de edad ${ }^{[29]}$.

Posteriormente aparecieron los resultados del estudio BENEFIT (BENznidazole Evaluation for Interrupting Trypanosomiasis, por sus siglas en inglés) [30], que parecían contradecir los anterior mencionado. Pero, debemos tener en cuenta que los grupos de pacientes diferían sustancialmente: los pacientes de la prueba no aleatorizada tenían una edad promedio de 39 años y en dos tercios de la población la función cardiaca de base era normal [14]. En contraste en la prueba BENEFIT la edad promedio de los participantes fue de 55 años, todos tenían daño cardiaco basal según hallazgos del electrocardiograma y casi la mitad una disminución de la fracción de eyección indicando disfunción ventricular ${ }^{[30,31]}$.

Por esto la pregunta de si el tratamiento proporciona algún beneficio clínico a los que no tengan compromiso cardiaco o aquellos con signos tempranos permanece sin responder ${ }^{[32,33]}$. Aunado a lo anterior y pese a la heterogeneidad de todas las pruebas clínicas, algunas revisiones muestran beneficio claro en cuanto a pruebas parasitológicas/serológicas, sin embargo, con resultados inconsistentes, pero con una tendencia a favorecer el tratamiento en este grupo de pacientes, en especial en los menores de 50 años y sin compromiso cardiaco establecido $[29,34,35]$.

Según datos de ensayos clínicos recientes, la monoterapia con benznidazole es una alternativa adecuada al tratamiento comparada con la terapia combinada con otros fármacos (posaconazole) en este grupo de pacientes ${ }^{[36]}$, en cuanto a la eliminación de la parasitemia en seguimiento hasta 12 meses; aunque hay algunos aspectos no claros como la evaluación de la disminución de la dosis de benznidazole en la terapia combinada para disminuir los eventos adversos y pero que conserven su eficacia.

Lo expuesto lleva a plantear la potencial posibilidad de tratamiento etiológico en ocho de los pacientes estudiados, con un buen grado de recomendación debido a que se encuentran en fase indeterminada y son menores de 50 años; existiendo menor consistencia en la indicación de tratar los restantes seis pacientes en igual fase pero que son mayores de 50 años.

En conclusión, los hallazgos de nuestro estudio son muy interesantes desde el punto de vista diagnóstico. El hallazgo en el índice de variabilidad de la frecuencia cardiaca se debería considerar como un posible indicador importante en esta patología, en cuanto al reconocimiento temprano antes de notar alteraciones estructurales que generen una falla cardiaca sintomática en esta patología.
Adicionalmente se debería establecer en pruebas clínicas prospectivas si el utilizar el ecocardiograma y monitoreo Holter para la caracterización de cardiopatía chagásica sería mejor que la definición con radiografía de tórax y electrocardiograma, pues estos tienen un mejor rendimiento en la identificación de alteraciones antes del desarrollo de cualquier síntoma y en especial anomalías en los últimos estudios (radiografía y electrocardiograma).

Lo anterior siempre en miras a ofrecer un posible tratamiento que pueda retrasar la aparición de una enfermedad sintomática por lo general con un compromiso cardiaco severo y de la fracción de eyección, sin embargo, como se ha mencionado con una discusión aun respecto a la utilidad de dicha medida en la fase indeterminada de la enfermedad.

Fuente de financiamiento: La fuente de financiación provino de recursos propios de los autores, sin recibir financiamiento por entidades externas ni públicas ni privadas.

Declaración de conflicto de intereses: Los autores declaran no tener conflicto de intereses con la publicación de este artículo.

\section{REFERENCIAS BIBLIOGRÁFICAS}

1. Rassi A, Jr., Rassi A, Marcondes de Rezende J. American trypanosomiasis (Chagas disease). Infect Dis Clin North Am. 2012;26(2):275-91.

2. Perez-Molina JA, Molina I. Chagas disease. Lancet. 2017. doi: 10.1016/S0140-6736(17)31612-4. [Epub ahead of print]

3. Cruz RE, Macedo AM, Barnabe C, Freitas JM, Chiari E, Veloso $V M$, et al. Further genetic characterization of the two Trypanosoma cruzi Berenice strains (Be-62 and Be-78) isolated from the first human case of Chagas disease (Chagas, 1909). Acta Trop. 2006;97(3):239-46.

4. Aufderheide AC, Salo W, Madden M, Streitz J, Buikstra J, Guhl F, et al. A 9,000-year record of Chagas' disease. Proc Natl Acad Sci U S A. 2004;101(7):2034-9.

5. Chagas disease (American trypanosomiasis) fact sheet (revised in June 2010). Wkly Epidemiol Rec. 2010;85(34):334-6.

6. Perez-Molina JA, Perez AM, Norman FF, Monge-Maillo B, LopezVelez R. Old and new challenges in Chagas disease. Lancet Infect Dis. 2015;15(11):1347-56.

7. Chagas disease in Latin America: an epidemiological update based on 2010 estimates. Releve epidemiologique hebdomadaire. 2015;90(6):33-43.

8. Dias E, Laranja FS, Miranda A, Nobrega G. Chagas' disease; a clinical, epidemiologic, and pathologic study. Circulation. 1956;14(6):1035-60.

9. Malik LH, Singh GD, Amsterdam EA. The Epidemiology, Clinical Manifestations, and Management of Chagas Heart Disease. Clin Cardiol. 2015;38(9):565-9.

10. Rassi A, Jr., Rassi A, Marin-Neto JA. Chagas disease. Lancet. 2010;375(9723):1388-402.

11. Martinez-Tovar JG, Rebollar-Tellez EA, Fernandez Salas I. Seroprevalence of T. cruzi infection in blood donors and Chagas cardiomyopathy in patients from the coal mining region of Coahuila, Mexico. Rev Inst Med Trop Sao Paulo. 2014;56(2):169-74. 
12. Sosa-Jurado F, Mazariego-Aranda M, Hernandez-Becerril N, Garza- Murillo V, Cardenas M, Reyes PA, et al. Electrocardiographic findings in Mexican chagasic subjects living in high and low endemic regions of Trypanosoma cruzi infection. Mem Inst Oswaldo Cruz. 2003;98(5):605-10.

13. Coura JR, de Abreu LL, Willcox HP, Petana W. [Comparative controlled study on the use of benznidazole, nifurtimox and placebo, in the chronic form of Chagas' disease, in a field area with interrupted transmission. I. Preliminary evaluation]. Rev Soc Bras Med Trop. 1997;30(2):139-44.

14. Viotti R, Vigliano C, Lococo B, Bertocchi G, Petti M, Alvarez $M G$, et al. Long-term cardiac outcomes of treating chronic Chagas disease with benznidazole versus no treatment: a nonrandomized trial. Ann Intern Med. 2006;144(10):724-34.

15. Chaves ÁM, Villar JC, Herrera M, Martínez LX, Cardona SR, Casadiego G. Estudio electrocardiográfico en sujetos con serología positiva y negativa para Tripanosoma Cruzi. Rev Col Cardiol 2004;11(5):246-50.

16. Sánchez SY, Velásquez TR, Bocangel BC, Vásquez HL, Córdova $B E$, Delgado DF, et al. Estudio comparativo de alteraciones electrocardiográficas, frecuencia cardiaca y presión arterial entre seropositivos y seronegativos para Trypanosoma cruzi en el valle de Vítor, Arequipa-Perú. Acta Med Per. 2007;24(2):7680 .

17. Navarro EC, Goto RL, Ricoboni IS, Corrente JE, Henriques RMS, Neves SL, et al. Seroprevalence of chagasic infection in young individuals in a blood center in the state of são paulo, brazil. Rev Inst Med Trop Sao Paulo. 2013;55(4):245-50.

18. Rigou D, Gullone N, Carnevali L, De rosa AF. chagas asintomatico hallazgos electrocardiograficos y ecocardiograficos. Medicina. 2001;61(5/1):541-4.

19. Botoni FA, Ribeiro AL, Marinho CC, Lima MM, Nunes Mdo C, Rocha MO. Treatment of Chagas cardiomyopathy. BioMed research international. 2013;2013:849504.

20. Andrade JP, Marin-Neto JA, Paola AA, Vilas-Boas F, Oliveira GM, Bacal $F$, et al. [I Latin American guidelines for the diagnosis and treatment of Chagas cardiomyopathy]. Arquivos brasileiros de cardiologia. 2011;97(2 Suppl 3):1-48.

21. Rassi A, Jr., Rassi A, Little WC, Xavier SS, Rassi SG, Rassi AG, et al. Development and validation of a risk score for predicting death in Chagas' heart disease. N Engl J Med. 2006;355(8):799-808.

22. Tanowitz HB, Morris SA, Factor SM, Weiss LM, Wittner M. Parasitic diseases of the heart I: Acute and chronic Chagas' disease. Cardiovasc Pathol. 1992;1(1):7-15.

23. Rocha MO, Ribeiro AL, Teixeira MM. Clinical management of chronic Chagas cardiomyopathy. Front Biosci. 2003;8:e44-54.

24. Ribeiro AL, Sabino EC, Marcolino MS, Salemi VM, Ianni BM, Fernandes $F$, et al. Electrocardiographic abnormalities in
Trypanosoma cruzi seropositive and seronegative former blood donors. PLoS Negl Trop Dis. 2013;7(2):e2078.

25. Nunes MC, Dones W, Morillo CA, Encina JJ, Ribeiro AL, Council on Chagas Disease of the Interamerican Society of C. Chagas disease: an overview of clinical and epidemiological aspects. J Am Coll Cardiol. 2013;62(9):767-76.

26. Ianni BM, Arteaga E, Frimm CC, Pereira Barretto AC, Mady C. Chagas' heart disease: evolutive evaluation of electrocardiographic and echocardiographic parameters in patients with the indeterminate form. Arq Bras Cardiol. 2001;77(1):59-62.

27. Arias E, Streiger M, Demonte MA, Del Barco M, Fabbro D, Ingaramo M. Alteraciones electrocardiográficas en preconscriptos con serología positiva y negativa para infección chagásica en áreas de la provincia de Santa Fe Rey Arg Cardiol. 1994;61(1):69-74.

28. Leon H, Guzman JC, Morillo CA. Alteraciones en la dinámica no lineal de la variabilidad de la frecuencia cardiaca en diferentes estadios de la enfermedad de chagas. Revista Iberoamericana de Arritmología 2009;1(1):48-56.

29. Bern C, Montgomery SP, Herwaldt BL, Rassi A, Jr., Marin-Neto JA, Dantas RO, et al. Evaluation and treatment of chagas disease in the United States: a systematic review. JAMA 2007;298(18):2171-81.

30. Morillo CA, Marin-Neto JA, Avezum A, Sosa-Estani S, Rassi A, Jr., Rosas F, et al. Randomized Trial of Benznidazole for Chronic Chagas' Cardiomyopathy. N Engl J Med. 2015;373(14):1295306.

31. Rassi A, Jr., Marin JAN, Rassi A. Chronic Chagas cardiomyopathy: a review of the main pathogenic mechanisms and the efficacy of aetiological treatment following the BENznidazole Evaluation for Interrupting Trypanosomiasis (BENEFIT) trial. Mem Inst Oswaldo Cruz. 2017;112(3):224-35

32. Maguire JH. Treatment of Chagas' Disease--Time Is Running Out. N Engl J Med. 2015;373(14):1369-70.

33. Bern C. A New Epoch in Antitrypanosomal Treatment for Chagas Disease. J Am Coll Cardiol. 2017;69(8):948-50.

34. Villar JC, Perez JG, Cortes OL, Riarte A, Pepper M, MarinNeto JA, et al. Trypanocidal drugs for chronic asymptomatic Trypanosoma cruzi infection. Cochrane Database Syst Rev. 2014(5):CD003463.

35. Perez-Molina JA, Perez-Ayala A, Moreno S, Fernandez-Gonzalez MC, Zamora J, Lopez-Velez R. Use of benznidazole to treat chronic Chagas' disease: a systematic review with a metaanalysis. J Antimicrob Chemother. 2009;64(6):1139-47.

36. Morillo CA, Waskin H, Sosa-Estani S, Del Carmen Bangher M, Cuneo C, Milesi R, et al. Benznidazole and Posaconazole in Eliminating Parasites in Asymptomatic T. Cruzi Carriers: The STOP-CHAGAS Trial. J Am Coll Cardiol. 2017;69(8):939-47. 